\title{
Aceitabilidade e composição centesimal de produtos alimentícios desenvolvidos com polpa de abacate
}

\section{Acceptability and centesimal composition of foods developed with avocado pulp}

\author{
Edilene do Nascimento Nunes ${ }^{1}$, Regina Célia Rodrigues de Miranda Milagres ${ }^{2}$, Cristiane \\ Gonçalves de Oliveira Fialho ${ }^{3}$, Eliana Carla Gomes de Souza ${ }^{4}$
}

\begin{abstract}
Resumo
O desenvolvimento de massa de pizza e flan com polpa de abacate é uma das estratégias na busca da prevenção de doenças crônicas não-transmissíveis, visto que o abacate é um produto com custo acessível e com alto valor nutricional. O objetivo foi avaliar a aceitabilidade e composição centesimal de produtos com polpa de abacate. Foi desenvolvida uma massa de pizza com adição de polpa de abacate e um flan com polpa de abacate e chia. Investigaram-se os teores de umidade, cinzas, proteínas, lipídios, carboidratos e calorias e aceitabilidade por meio de análise sensorial. A massa de pizza com abacate apresentou-se menos calórica e com quantidades inferiores de macromoléculas comparadas ao controle. Com relação aos atributos sensoriais obteve boa aceitabilidade na cor e textura, porém o sabor e a impressão global receberam notas 5,7 e 6,2, respectivamente. A quantidade de calorias do flan foi inferior a encontrada nas sobremesas disponíveis no comércio. A preparação obteve boa aceitação em todos os parâmetros avaliados, com valores em torno de 7 (gostei moderadamente). Conclui-se que a massa de pizza com abacate necessita de mais estudos para melhorar o sabor residual amargo, no entanto, o flan tem potencial para ser oferecido no mercado em função de seu valor nutricional e de sua boa aceitação.
\end{abstract}

Palavras chave: Análise sensorial. Composição química. Desenvolvimento de produtos.

\begin{abstract}
Given that the avocado is a product with affordable cost and high nutritional value, the development of pizza and flan pasta with avocado pulp is one of the strategies in the search of preventive resources for chronic non-transmitted diseases. The objective of the study was to evaluate the acceptability and centesimal composition of products with avocado pulp. A pizza dough was developed with avocado pulp and a flan was made with avocado pulp and chia. The investigation focused on the levels of moisture, ashes, proteins, lipids, carbohydrates and calories and the acceptability through sensorial analysis. The pizza dough with avocado was less caloric and contained lower amounts of macromolecules in comparison to the control. Regarding the sensorial attributes, it obtained good acceptability in color and texture, however, the taste and the overall impression received 5.7 and 6.2, respectively. The amount of calories in the flan was inferior than those found in commercially available desserts. The preparation was well accepted in all evaluated parameters, with values around 7 (I moderately liked it). It is concluded that the pizza dough with avocado needs further studies to improve the residual bitter taste, conversely, the flan has the potential to be offered in the market due to its nutritional value and good acceptance.
\end{abstract}

Keywords: Sensory analysis. Chemical composition. Product development.

\footnotetext{
${ }^{1}$ Mestranda em Nutrição pela Universidade Federal de Ouro Preto

${ }^{2}$ Doutora em Ciências pela Universidade de São Paulo, São Paulo, Brasil. Técnico de Nível Superior. Departamento de Nutrição e Saúde da Universidade Federal de Viçosa, Viçosa, Minas Gerais, Brasil. E-mail: reginamilagres@ufv.br

${ }^{3}$ Doutora em Ciência da Nutrição pela Universidade Federal de Viçosa, Viçosa, Minas Gerais, Brasil. Professora do Departamento de Nutrição da Universidade Federal de Juiz de Fora, Juiz de Fora, Minas Gerais, Brasil.

${ }^{4}$ Doutora em Ciência e Tecnologia de Alimentos pela Universidade Federal de Viçosa, Viçosa, Minas Gerais, Brasil. Professora do Departamento de Nutrição e Saúde da Universidade Federal de Viçosa, Viçosa, Minas Gerais, Brasil.
} 


\section{Introdução}

O estilo de vida da população brasileira tem sido marcado pela diminuição no gasto energético, alimentação advinda de produtos industrializados, ricos em açúcar e gorduras, além da redução do consumo de carboidratos complexos e fibras. Essa forma de se alimentar, aliada ao sedentarismo crescente traz como consequência às doenças crônicas não transmissíveis (DCNT). ${ }^{(1)}$

As DCNT são as principais causas de mortes no Brasil. Em 2009 representaram 72,4\% dos óbitos. ${ }^{(2)}$ Além disso, cerca de $20 \%$ de todas as mortes em pessoas acima de 30 anos são por doenças cardiovasculares. ${ }^{(3)}$

Estudos comprovam que o consumo de dietas ricas em ácidos graxos monoinsaturados, principalmente o ácido oléico, substituindo os saturados em humanos, reduz as concentrações de colesterol total, triacilglicerídeos e LDL-colesterol, que são os principais fatores de risco para o desenvolvimento de doenças cardiovasculares. Alimentos fontes destes ácidos graxos são o azeite e as oleaginosas, entretanto, estes alimentos não são acessíveis a toda população por seu elevado custo. ${ }^{(4)} \mathrm{O}$ abacate é uma boa opção para substituir esses alimentos. Encontra-se entre os frutos mais vendidos no mundo, principalmente, por seu custo acessível e produção em quase todos os meses do ano. É um fruto subtropical e tropical com $70 \%$ de polpa, onde $100 \mathrm{~g}$ in natura possui em média $17,34 \mathrm{~g}$ de lipídios. Esta fração lipídica tem propriedade funcional visto que é rica em ácidos graxos monoinsaturados, fitoesteróis e tocoferóis. Estes são benéficos à saúde, auxiliando na prevenção de doenças cardiovasculares, uma vez que ajudam na redução dos níveis séricos de colesterol total, colesterol LDL e triacilglicerídeos, e promovem o aumento do colesterol HDL. ${ }^{(5)}$

Diante isso, o desenvolvimento de produtos com polpa de abacate com a finalidade de substituir óleos com baixa quantidade de monoinsaturados ou de alto preço é um campo de pesquisa a ser explorado, principalmente, com a transição nutricional atual. A grande oferta de produtos populares pobres em micronutrientes e ricos em gorduras saturadas e açúcares simples é um incentivo à busca de adaptações. Escolheu-se pizza e flan por serem bem aceitos pela população brasileira, sendo essas adaptadas para melhorar a qualidade nutricional.

O objetivo foi analisar a composição centesimal e a aceitabilidade de produtos elaborados com polpa de abacate, a fim de produzir alimentos com maior valor nutricional e aceitáveis sensorialmente.

\section{Material e método}

O desenvolvimento de formulações de massa de pizza e flan com polpa de abacate (Persea americana) ocorreu no Laboratório de Estudo Experimental dos Alimentos do Departamento de Nutrição e Saúde da Universidade Federal de Viçosa.

\section{Formulações:}

\section{Massa de Pizza:}

Com abacate: ingredientes - $800 \mathrm{~g}$ de farinha de trigo; $300 \mathrm{~mL}$ de água morna, $200 \mathrm{~g}$ da polpa do abacate, $5 \mathrm{~g}$ de açúcar e $5 \mathrm{~g}$ de fermento seco.

Sem abacate: ingredientes - os mesmos da preparação com abacate, substituindo-se a polpa do abacate por $75 \mathrm{~mL}$ de azeite de oliva extra virgem.

Para análise de semelhança entre os lipídeos da preparação optou-se pelo azeite de oliva que é fonte de ômega 3, 6 e 9, assim como abacate, logo utilizouse uma média padrão da quantidade de lípidios que haveria na polpa de abacate e chegou-se nessa proporção de $20 \%$ de polpa para $80 \%$ de farinha.

Para o preparo, os ingredientes secos foram misturados e adicionados ao fermento previamente diluído em água morna. A massa foi sovada até ficar uniforme e permaneceu em repouso por $60 \mathrm{~min}$. Em seguida a massa foi moldada em formato de pizza e assada em forno pré-aquecido por $10 \mathrm{~min}$, a $220^{\circ} \mathrm{C}$.

\section{Flan de Abacate:}

Ingredientes - $900 \mathrm{~g}$ de polpa de abacate; $450 \mathrm{~mL}$ de leite integral; $250 \mathrm{~mL}$ de água; $165 \mathrm{~g}$ de açúcar; $20 \mathrm{~g}$ de semente de chia e $12 \mathrm{~g}$ de gelatina sem sabor. Os ingredientes utilizados no preparo do Flan foram previamente separados e pesados. A gelatina foi hidratada, conforme instruções da embalagem. O gel de chia foi elaborado com $200 \mathrm{~mL}$ de água e $20 \mathrm{~g}$ de chia e aquecidos até engrossar. A polpa de abacate foi adicionada à gelatina e ao gel de chia e homogeneizados com um Mixer. A emulsão resultante foi dispensada em copos descartáveis e refrigerada por cerca de $6 \mathrm{~h}$.

Foram realizadas análises de Composição Centesimal das formulações conforme descrito a seguir:

Massa de Pizza: a composição química das massas de pizzas foi realizada por métodos bromatológicos $^{(6)}$ no Laboratório de Análise 
Instrumental de Alimentos da Universidade Federal de Juiz de Fora.

- Umidade: método gravimétrico, por meio da secagem em estufa a $105^{\circ} \mathrm{C}$.

- Cinzas: método gravimétrico, por aquecimento a $550^{\circ} \mathrm{C}$ em mufla.

- Proteínas: método de Kjeldahl, por meio de digestão com $\mathrm{H}_{2} \mathrm{SO}_{4}$, destilação com $\mathrm{NaOH}$ $50 \%$ e titulação com $\mathrm{H}_{2} \mathrm{SO}_{4} 0,02 \mathrm{~N}$. Para a conversão do nitrogênio em proteína, utilizouse o fator 6,25 .

- Lipídeos: extração com éter de petróleo, por 4 horas, em aparelho do tipo Soxhlet.

- Carboidratos Totais: determinados por diferença entre 100 e a soma dos teores de umidade, cinzas, lipídios e proteínas.

- Calorias: o valor calórico total foi calculado a partir da soma das calorias correspondentes para proteínas, lipídeos e carboidratos.

Flan: A composição química dos nutrientes foi realizada por meio de consulta em tabelas de composição química de alimentos, disponíveis na literatura como a Tabela Brasileira de Composição de alimentos ${ }^{(7)}$ e Tabela de composição de alimentos: suporte para decisão nutricional. ${ }^{(8)} \mathrm{A}$ composição química dos alimentos industrializados foi obtida dos rótulos.

Análises sensoriais da massa de pizza e do flan foram realizadas no Laboratório de Análise Sensorial dos Alimentos no Departamento de Nutrição e Saúde, da Universidade Federal de Viçosa.

O teste de aceitação dos produtos foi realizado em cabines individuais onde cada julgador assinou o Termo de Consentimento Livre e Esclarecido com informações sobre o estudo. Após assinar o termo, o voluntário recebeu duas amostras de massa de pizza e uma de flan codificadas com números de três dígitos aleatórios e um copo de água para a limpeza das papilas entre as degustações. Receberam também uma ficha de avaliação da aceitabilidade das massas de pizza e do flan com escala hedônica de 9 pontos, em que: $1=$ desgostei extremamente; $5=$ não gostei nem desgostei e 9= gostei extremamente para os atributos: sabor, cor, textura e aparência global..$^{(9)}$

$O$ teste foi realizado com 50 julgadores não treinados, entre estudantes, professores e servidores da UFV, de ambos os sexos. Os indivíduos foram informados a respeito de sua participação voluntária por meio de um Termo de Consentimento Livre e
Esclarecido (TCLE), constando os dados da pesquisa.

Foram excluídos os indivíduos que não assinaram o TCLE e também aqueles que não preencheram corretamente a ficha de avaliação.

$O$ índice de aceitabilidade das formulações foi calculado conforme fórmula: IA (\%) $=\mathrm{A} \times 100 / \mathrm{B}$, onde $\mathrm{A}=$ nota média obtida para o produto; $\mathrm{B}=$ nota máxima dada ao produto.

Antes de qualquer abordagem o estudo foi aprovado pela Comissão de Ética e experimentação com seres humanos da Universidade Federal de Viçosa (UFV), sob parecer de número 474.773 .

Os resultados foram submetidos à análise de variância, de acordo com os procedimentos estabelecidos no software Assistat, versão 7.7, para a verificação de diferenças estatisticamente significativas entre as médias das variáveis estudadas. Para a comparação entre as médias, empregou-se o teste de Tukey, adotando-se, como nível de significância, $a=0,05$.

\section{Resultados e Discussão}

Os resultados das análises da composição centesimal da massa de pizza e do flan, encontram-se na Tabela 1.

Pode-se observar na tabela 1 que a pizza com

Tabela 1 - Composição química das massas de pizza e do flan $\left(\mathrm{g} 100 \mathrm{~g}^{-1}\right)$.

\begin{tabular}{ccccccc}
\hline Formulações & Umidade & Cinzas & Lipídeos & Proteínas & Carboidratos & Calorias \\
\hline $\begin{array}{c}\text { Pizza sem } \\
\text { abacate }\end{array}$ & $13,43^{\mathrm{b}}$ & $1,00^{\mathrm{a}}$ & $9,34^{\mathrm{a}}$ & $5,06^{\mathrm{a}}$ & $71,17^{\mathrm{a}}$ & $389^{\mathrm{a}} \mathrm{kcal}$ \\
$\begin{array}{c}\text { Pizza com } \\
\text { abacate }\end{array}$ & $19,10^{\mathrm{a}}$ & $1,01^{\mathrm{a}}$ & $9,08^{\mathrm{b}}$ & $2,92^{\mathrm{b}}$ & $67,89^{\mathrm{b}}$ & $365^{\mathrm{b}} \mathrm{kcal}$ \\
Flan & 77,60 & 1,00 & 5,40 & 1,60 & 14,40 & $112,6 \mathrm{kcal}$
\end{tabular}

*Médias seguidas pela mesma letra na coluna não diferem entre si (Tukey, $\mathrm{p}<0,05$ ).

abacate apresentou quantidades de lipídeos, proteínas e carboidratos inferiores à massa controle, o que pode estar associado à adição de maior quantidade de abacate $(200 \mathrm{~g})$ do que de azeite $(75 \mathrm{~mL})$, diluindo assim, a concentração desses nutrientes na massa. Consequentemente, a quantidade calórica da pizza com abacate foi inferior, o que é benéfico, considerando que pizza é um produto muito consumido por crianças 
e adolescentes e a maior ingestão calórica pode levar à obesidade. O teor da umidade da pizza com abacate foi superior ao da amostra controle, provavelmente, em função da umidade proveniente do fruto.

De acordo com a recomendação da $\mathrm{RDC} \mathrm{n}^{\circ} 359$ de $2003^{(10)}$, a porção de massa de pizza deve corresponder a $150 \mathrm{Kcal}$, o que representa $38,5 \mathrm{~g}$ de massa sem abacate e $41 \mathrm{~g}$ de massa com abacate (Tabela 1 ).

São mais comuns no mercado as massas de pizza que em sua formulação é utilizada gordura hidrogenada (trans) com o objetivo de prolongar as características sensoriais e sua vida útil. Essa gordura trans atua no corpo como a gordura saturada, elevando os níveis de LDL, colesterol e, como consequência, riscos de desenvolver doenças cardiovasculares. Associado ao consumo de gordura trans, há a inibição do metabolismo de ácidos graxos essenciais. ${ }^{(11)}$

Os resultados obtidos na análise sensorial das pizzas demonstraram que não houve diferença na cor e na textura, o que é de grande importância para melhorar a aceitação do consumidor em um primeiro contato com o produto, além de indicar que a adição da polpa de abacate não interfere na consistência da mesma (Tabela 2).

Entretanto, a avaliação do sabor diferiu da amostra controle e, por consequência, reduziu a nota de Impressão Global (Tabela 2). Esses achados podem estar relacionados ao sabor amargo do abacate após aquecimento. Esse "off-flavor" é resultante da combinação de algumas substâncias químicas presentes no abacate e outras derivadas do aquecimento do mesmo. ${ }^{(12)}$ Esses autores identificaram 1-acetoxi-2,4-di-hidroxi-n-heptadeca-16-em e 1,2,4-trihidroxi-n-heptadeca-16-em em polpa de abacate aquecida e Bates ${ }^{(13)}$ encontrou feofitinas, que é um produto de degradação da clorofila, responsável pela coloração marrom-azeitona e sabor amargo. A reação de feofitinização ocorre quando, em meio ácido, o magnésio do centro da molécula de clorofila é substituído por hidrogênio. Para minimizar esse processo pode-se basificar o meio, o que torna a clorofila mais estável ao calor. ${ }^{(14)}$ No entanto, a adição de substâncias básicas nos alimentos pode levar à perdas de algumas vitaminas.

O flan de abacate apresentou 112,6 kcal, quantidade inferior a de outros produtos similares que, normalmente, possuem em torno de $128 \mathrm{kcal}$. Para atender a RDC no 359 de $2003^{(10)}$, que preconiza 125 kcal para uma porção de sobremesas lácteas, o flan deverá conter $111 \mathrm{~g}$ por porção.
Vale ressaltar, a boa qualidade nutricional do flan devido ao conteúdo de lipídeos advindos do abacate. Além disso, a chia adicionada possibilitou o aumento da concentração de ácidos graxos polinsaturados, especialmente o ômega $3 .^{(15)}$

Os ácidos graxos ômega 3, melhoram ainda mais o perfil lipídico dos produtos, além de possuir efeito anti-inflamatório. ${ }^{(16)}$

Tabela 2 - Teste de aceitação e índice de aceitabilidade (IA) das massas de pizza e do Flan.

\begin{tabular}{lccc}
\hline \multicolumn{1}{c}{ Avaliações } & $\begin{array}{c}\text { Pizza com } \\
\text { abacate }\end{array}$ & $\begin{array}{c}\text { Pizza sem } \\
\text { abacate }\end{array}$ & Flan \\
\hline $\begin{array}{l}\text { Cor (Média } \pm \text { Desvio } \\
\text { padrão) }\end{array}$ & $7,2 \pm 1,30^{\mathrm{a}}$ & $7,4 \pm 1,19^{\mathrm{a}}$ & $7,0 \pm 1,85$ \\
IA (\%) & 80 & 82 & 77 \\
$\begin{array}{l}\text { Textura (Média } \pm \text { Desvio } \\
\text { padrão) }\end{array}$ & $6,3 \pm 1,82^{\mathrm{a}}$ & $6,9 \pm 1,68^{\mathrm{a}}$ & $7,0 \pm 1,73$ \\
$\begin{array}{l}\text { IA (\%) } \\
\text { Sabor (Média } \pm \text { Desvio } \\
\text { padrão) }\end{array}$ & 70 & 77 & 78 \\
IA (\%) & $5,7 \pm 2,00^{\mathrm{b}}$ & $7,4 \pm 1,23^{\mathrm{a}}$ & $7,4 \pm 1,61$ \\
$\begin{array}{l}\text { Impressão Global } \\
\text { (Média } \pm \text { Desvio padrão) }\end{array}$ & $6,2 \pm 1,61^{\mathrm{b}}$ & $7,4 \pm 1,05^{\mathrm{a}}$ & $7,4 \pm 1,38$ \\
IA (\%) & 69 & 82 & 82 \\
\hline $\begin{array}{l}\text { Médias seguidas pela mesma letra na linha não diferem entre si (Tukey, } \\
\text { p }<0,05) .\end{array}$ & \multicolumn{2}{|}{}
\end{tabular}

Percebe-se que o flan obteve boa aceitação em todos os atributos e alto índice de aceitabilidade (IA), o que indica possível aceitação no mercado (Tabela 2). Esta sobremesa foi desenvolvida pela facilidade de preparo, baixo custo e forte apelo nutricional, por conter pouco açúcar e adição de chia e abacate, o que até o momento não foi encontrado na literatura. A principal finalidade, neste estudo, foi demonstrar que uma sobremesa com valor nutricional agregado pode ter boa aceitabilidade, conforme demonstrado nas análises sensoriais. Estes resultados indicam que é um produto com forte capacidade mercadológica.

Em estudo de Soler et al., ${ }^{(17)}$ sobremesas lácteas achocolatadas preparadas com $28,8 \%, 36,3 \%$ e $41,7 \%$ de polpa de abacate apresentaram IA de $81 \% ; 73 \%$ e $53 \%$, respectivamente. O flan elaborado no presente estudo continha $50 \%$ de abacate e ainda assim apresentou IA de $82 \%$, o que é maior do que todas as formulações apresentadas no estudo supracitado.

O abacate possui compostos com capacidade antioxidante com teores significativos em todo fruto. ${ }^{(18)}$ Os compostos antioxidantes trazem benefícios à 
saúde. Os benefícios destes compostos também foram comprovados no combate a infecção por Helicobacter pylori.

O uso de polpa de abacate como ingrediente em preparações, apresenta-se como uma excelente alternativa, visto que seu conteúdo de ácidos graxos monoinsaturados traz benefícios comprovados. Dentre eles estão a redução dos níveis de colesterol sanguíneo, hepático e LDL, além de aumentar o colesterol excretado e manter os níveis de HDL e triglicerídeos estáveis. ${ }^{(4)}$

É urgente a necessidade de redução do consumo de produtos industrializados, de baixa qualidade nutricional e nocivos à saúde. Assim, alimentos alternativos que auxiliem na redução do consumo destes produtos são fundamentais. Diante disso, é necessário que a formulação da massa de pizza seja ajustada de forma a retirar ou reduzir o sabor residual, uma vez que, pizza é um produto muito consumido e aceito pela população e seus benefícios irão contribuir para melhorar o valor nutricional da alimentação. Acredita-se que o flan já pode ser oferecido aos consumidores, pois, apresentou alta aceitabilidade e possui valor calórico reduzido em comparação a outras sobremesas.

\section{Conclusões}

A massa de pizza elaborada com polpa de abacate obteve menor quantidade de calorias e boa aceitabilidade nos atributos cor e textura, porém ainda é preciso mais estudos para reduzir o sabor residual amargo deixado pelo abacate quando aquecido. O flan apresentou-se menos calórico do que os disponíveis no mercado e a boa aceitabilidade nos parâmetros de cor, sabor, textura e impressão global sugerem que é possível introduzi-lo no mercado. Considerando que, os lipídios do fruto de abacate são constituídos, principalmente, por ácidos graxos monoinsaturados e o abacate tem custo mais baixo que outros alimentos fontes destes compostos, infere-se que os produtos desenvolvidos neste estudo são uma excelente alternativa de alimento para o consumidor.

\section{Referências}

1 Souza EB. Transição nutricional no Brasil: análise dos principais fatores. Cad. UniFOA. 2010 ago;13:4953.
2 Duncan BB, Chor D, Aquino EML, Bensenor IM, Mill JG, Schmidt MI, et al. Doenças crônicas não transmissíveis no Brasil: prioridade para enfrentamento e investigação. Rev. Saúde Pública. 2012 dez;46(S 1):126-134. doi: 10.1590/S003489102012000700017

3 Mansur AP, Favarato D. Mortalidade por doenças cardiovasculares no Brasil e na região metropolitana de São Paulo: atualização 2011. Arq. Bras. Cardiol. 2012 ago;99(2):755-761. doi: 10.1590/S0066$782 \mathrm{X} 2012005000061$

4 Salgado JM, Bin C, Mansi DN, Souza A. Efeito do abacate (Persea americana Mill) variedade hass na lipidemia de ratos hipercolesterolêmicos. Ciênc. Tecnol. Aliment. 2008 out-dez;28(4):922-928. doi: 10.1590/S0101-20612008000400025

5 Chaves MA, Mendonça CRB, Borges CD, Porcu OM. Elaboração de biscoito integral utilizando óleo e farinha da polpa de abacate. B. CEPPA, 2013 juldez;31(2):215-226.

6 Association of Official Analytical Chemists. Official methods of analysis of AOAC International. $20^{\text {th }}$ ed. Rockville: AOAC; 2016.

7 Núcleo de Estudos e Pesquisas em Alimentação. Tabela Brasileira de Composição de Alimentos TACO. 4. ed. Campinas: UNICAMP; 2011.

8 Philippi ST. Tabela de composição de alimentos: suporte para decisão nutricional. 2. ed. São Paulo, Coronário; 2002.

9 Poste L, Mackie D, Butler G, Lamard E. Laboratory methods for sensory analysis of food. Ottawa: Agriculture Canada; 1991.

10 Ministério da Saúde (BR). Resolução RDC n. 359, de 23 de dezembro de 2003. A Diretoria Colegiada da ANVISA/MS aprova o regulamento técnico de porções de alimentos embalados para fins de rotulagem nutricional. Diário Oficial da União $26 \mathrm{dez}$ 2003; Seção 1. 
11 Calderelli VAS, Benassi MT, Matioli G. Substituição da gordura hidrogenada por óleo de soja na elaboração de pães de linhaça e avaliação da aceitabilidade. Ciênc. Tecnol. Aliment. 2008;28:668-674.

12 Ben-Et G, Dolev A, Tatarsk D. Compounds contributing to heatinduced bitter off-flavor in avocado. J. Food Sci.1973;38:546-547.

13 Bates RP. Heat-induced off-flavor in avocado flesh. J. Food Sci. 1970;35(4):478-482.

14 Von Elbe JH. Colorantes. In: Fennema OW. Química de los alimentos. 2. ed. Zaragoza: WisconsinMadison; 2000. p. 782-799.

15 Asif M. Health effects of omega-3, 6, 9 fclorofilidaatty acids: Perilla frutescens is a good example of plant oils. Orient Pharm Exp Med. 2011 Mar;11(1):51-59.

16 Mesquita TR, Souza AA, Constantino E, Pelógia NCC, Posso IP, Pires OC. Efeito anti-inflamatório da suplementação dietética com ácidos graxos ômega-3, em ratos. Rev. Dor. 2011;12(4):337-341. doi: $10.1590 / \mathrm{S} 1806-00132011000400010$

17 Soler N, Batista AG, Faria CAM, Gonzaga DG, Lopes JMM, Pinto NAVD. Elaboração, composição química e avaliação sensorial de sobremesas lácteas achocolatadas com abacate. Alim. Nutr. 2011 janmar; 22(1):143-148.

18 Daiuto ER, Tremocoldi MA, Alencar SM, Vieites RL, Miet PH. Composição química e atividade antioxidante da polpa e resíduos de abacate 'hass. Rev. Bras. Frutic. 2014 abr;36(1):417-424. 